Please do not remove this page

RMIT

UNIVERSITY

\title{
Adaptive modulation with space-time block coding for MIMO-OFDM systems
}

Lin, Kevin; Mahmoud, Seedahmed; Hussain, Zahir

https://researchrepository.rmit.edu.au/esploro/outputs/9921858657501341/filesAndLinks?institution=61RMIT_INST\&index=null

Lin, K., Mahmoud, S., \& Hussain, Z. (2005). Adaptive modulation with space-time block coding for MIMO-OFDM systems. Proceedings of the Third International Conference on Information Technology and Applications, 299-304. https://doi.org/10.1109/ICITA.2005.53

Published Version: https://doi.org/10.1109/ICITA.2005.53

Repository homepage: https://researchrepository.rmit.edu.au

(c) 2005 IEEE. Personal use of this material is permitted. However, permission to reprint/republish this material for advertising or promotional purposes or for creating new collective works for resale or redistribution to servers or lists, or to reuse any copyrighted component of this work in other works must be obtained from the IEEE.

Downloaded On 2023/04/26 23:16:02 +1000 


\title{
Adaptive Modulation with Space-Time Block Coding for MIMO-OFDM Systems
}

\author{
Kevin H. Lin, Seedahmed S. Mahmoud, and Zahir M. Hussain \\ School of Electrical and Computer Engineering, RMIT University \\ 124 LaTrobe Street, Melbourne, Victoria 3000, Australia \\ s9510490@student.rmit.edu.au; mahmoud.seedahmed@rmit.edu.au; zmhussain@ieee.org
}

\begin{abstract}
Space-time block coding (STBC) scheme has proved to be efficient in enhancing the performance of communication systems in terms of both bit-error-rate (BER) and symbol-error-rate (SER). Adaptive bit and power allocation (ABPA), on the other hand, is an adaptive modulation scheme that assigns an optimum symbol constellation and power to all subcarriers in a multi-carrier system to improve spectral and power efficiency. In this work, we combine a lookup matrix based ABPA algorithm with subchannel grouping (LM-SG-ABPA) and STBC for orthogonal frequency division multiplexing (OFDM) systems to further enhance the performance and support high data rate applications. Numerical results showed that the proposed transmission structure achieves not only a significantly higher spectral and power efficiency over systems that do not employ any ABPA schemes, but also a better error probability performance in a multiple-input multiple-output (MIMO) channel.
\end{abstract}

\section{Introduction}

Increasing demands for high spectral efficiency and reliable communication revealed the need for advanced transmission techniques in multiple-input multiple-output (MIMO) channels [1]-[3]. In particular, space-time coding [3] demonstrated that it can greatly improve system capacity and error rate performance when data symbol energy is spread across space and time. This performance improvement, however, came with dramatic increase in coding and decoding complexity. Space-time block coding (STBC) was then later introduced in [4] to mitigate this computational load and still retain much of the performance gain.

To enhance the spectral efficiency of band limited systems, various adaptive multicarrier modulation schemes, namely adaptive bit and power allocation (ABPA), have been reported in [5] - [7]. Their proposed allocation algorithms are, however, either computationally inefficient or impractical due to slow convergence speed.

In this paper, we introduce a transmission structure that incorporates an algorithmically efficient LM-SG-ABPA scheme in [8] with STBC (called LM-SG-ABPA-STBC) for MIMO-OFDM systems. By knowing the sub-channel gain, the proposed configuration first constructs a lookup matrix based on a target BER constraint. Then it identifies subcarriers that require the least amount of power for transmitting an additional data bit and perform a bit-by-bit allocation scheme across the subcarriers. The resultant effect of this adaptive modulation scheme is the optimum allocation of data rate and power across subcarrier so that maximum spectral efficiency is achieved without wasting power or sacrificing BER. To reduce the size of the lookup matrix and amount of feedback signaling bandwidth, the concept of sub-channel grouping is utilized. The adaptively modulated output symbols are then space-time block encoded within each subcarrier before transmitting on the antennas for further improving the error rate performance.

Notation used in this paper: $(.)^{T}$, and $(.)^{H}$ are vector, and Hermitian transposition respectively. $\|\mathcal{A}\|_{F}$ denotes the Frobenius norm of matrix $\mathcal{A}$. Finally, capital bold letters represent matrices, and small underlined letters are vectors.

\section{System Model}

Consider a MIMO-OFDM wireless communication system employing $N_{t}$ transmit and $N_{r}$ receive antennas. We assume that the system is wideband and $N_{c}$ orthogonal frequency subcarriers are utilized. We also assume that $N_{c}$ is large enough so that each subcarrier can be considered as narrowband. In general, broadband OFDM signal propagation experiences frequency-selective fading and causes subcarriers to attenuate differently. Let us define the channel impulse response vector between the $i^{\text {th }}$ transmit and the $j^{\text {th }}$ receive antenna as $\underline{h}_{i, j}=\left[h_{i, j}(1), \cdots, h_{i, j}(L-1)\right]$ with $L$ denoting the channel order. In this work, impulse response vector elements are modelled as complex Gaussian random variables having Jakes power spectral density. A general structure of this OFDM system combined with 
the proposed LM-SG-ABPA scheme and STBC is shown in Fig. 1, which we will specify next.

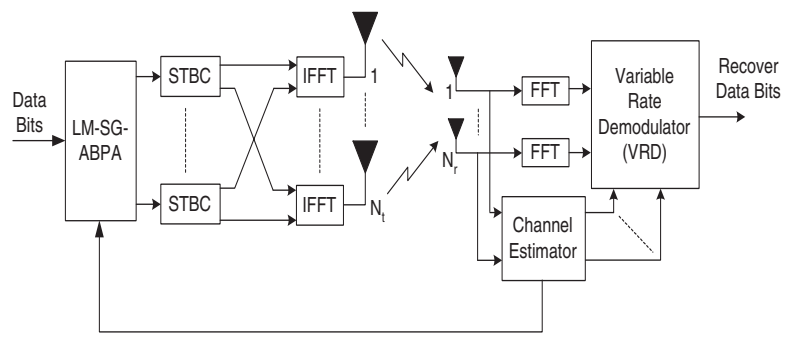

Figure 1. General structure of proposed
OFDM transmission scheme employing adap-
tive modulation and space-time block coding.

At the transmitter, $N_{s}$ information bits are first serial-toparallel converted into $N_{c}$ frequency subcarriers before the LM-SG-ABPA adaptive modulation process. Based on the channel estimates in the receiver feedback message, different baseband signal constellation size is used and power is allocated for different subcarriers during the LM-SG-ABPA process. Therefore, the number of information bits transmitted varies from one subcarrier to another. Denote $\rho_{k}$ as the amount of power allocated to the $k^{\text {th }}$ subcarrier and let the commonly used notation $\left\{M_{k} \mid k=1, \cdots, N_{c}\right\}$ be the assigned signal constellation for OFDM subcarriers. Hence $\omega_{k}=\log _{2} M_{k}$ is the number of information bits assigned to the $k^{\text {th }}$ subcarrier, i.e. $2^{\omega_{k}}-$ ary PSK or $2^{\omega_{k}}-$ ary QAM, and $N_{s}=\sum_{k=1}^{N_{c}} \omega_{k}$. The detailed of LM-SG-ABPA process will be describe in Section 3.

Since data symbols are ST block encoded in the proposed transmission structure, we regard all signal transmissions under consideration here as block transmissions. In the well-known STBC of [4], a different ST block encoding matrix requires different number of input data symbols for different number of transmit antennas. We assume that the space-time block encoding matrix $\mathcal{G} 4$

$$
\mathcal{G} 4=\left(\begin{array}{rrrr}
s_{1} & s_{2} & s_{3} & s_{4} \\
-s_{2} & s_{1} & -s_{4} & s_{3} \\
-s_{3} & s_{4} & s_{1} & -s_{2} \\
-s_{4} & -s_{3} & s_{2} & s_{1} \\
s_{1}^{*} & s_{2}^{*} & s_{3}^{*} & s_{4}^{*} \\
-s_{2}^{*} & s_{1}^{*} & -s_{4}^{*} & s_{3}^{*} \\
-s_{3}^{*} & s_{4}^{*} & s_{1}^{*} & -s_{2}^{*} \\
-s_{4}^{*} & -s_{3}^{*} & s_{2}^{*} & s_{1}^{*}
\end{array}\right)
$$

is employed for systems with $N_{t}=4$. In this case, four input data symbols per subcarrier is required for ST block encoding and the signal (block) transmission of this matrix will take over eight OFDM-symbol length to complete.
Thus, block encoding rate of $\mathcal{G} 4$ is 0.5 and the data transmission rate of the system becomes $0.5 \sum_{k=1}^{N_{c}} \omega_{k}$. If $\mathcal{G} 2$ is used instead with $N_{t}=2$, then it will incur no rate loss.

Let $\mathbf{X}_{k}$ be an encoded matrix at the output of space-time block encoder and transmitted on the $k^{\text {th }}$ subcarrier using $N_{t}$ transmit antennas over the frequency-selective channel. Note that the difference between $\mathbf{X}_{k}$ and $\mathbf{X}_{\ell} \forall \ell \neq k$ is not only the information data that matrices are containing but also the modulating constellation used and power that is allocated. At the receiver, suppose that discrete Fourier transformation is applied to the received signals from $N_{r}$ antennas. Then the discrete time baseband equivalent expression of the received signal will be

$$
\mathbf{Y}_{k}=\sqrt{\frac{\rho_{k}}{N_{t}}} \mathbf{X}_{k} \mathbf{H}_{k}+\mathbf{E}_{k}
$$

where $\mathbf{E}_{k}$ is a additive Gaussian noise matrix at the receiver with each element having zero mean and $\sigma_{k}^{2}$ variance. $\mathbf{H}_{k} \in \mathbb{C}^{N_{t} \times N_{r}}$ is the channel frequency response matrix with its $(i, j)^{\text {th }}$ entry $\mathbf{H}_{k}(i, j)=\underline{h}_{i, j} \underline{f}_{k}$ and $\underline{f}_{k}=$ $\left[1, e^{-j 2 \pi(k-1) / N_{c}}, \cdots, e^{-j 2 \pi(k-1) \tau_{L-1} / N_{c}}\right]^{T} \in \mathbb{C}^{L \times 1}$ is the corresponding discrete Fourier transform coefficients.

At the receiver, channel estimation is performed by evaluating pilot tones embedded in the transmitted signal, then it is fed into the variable rate demodulator (VRD). The Internal structure of VRD consists of a maximum ratio combiner (MRC), a maximum likelihood detector (MLD) for decoding of STBC data symbols and a channel decoder for baseband demodulation of different signal constellation size used across subcarriers. Clearly, the receiver performs reverse functions of transmitter structure. Hence, the decision matrix evaluating at the MLD for the detection of $\mathbf{X}_{k}$ has the following form

$$
\widehat{\mathbf{X}}_{k}=\arg \min _{\mathbf{X}_{k}}\left\|\mathbf{Y}_{k}-\sqrt{\frac{\rho_{k}}{N_{t}}} \mathbf{X}_{k} \mathbf{H}_{k}\right\|_{F}^{2} .
$$

\section{LM-SG-ABPA Algorithm}

In this paper, the main objective of LM-SG-ABPA scheme is to maximize system throughput regardless of the block encoding rate of the STBC matrix that is used. However, this should be done subject to a total transmit power constraint while achieving a target BER performance on each subcarrier. Let us denote $B E R_{\text {tar }}$ to be the target BER, $2^{\omega_{k}}$ to be the symbol constellation size and $\rho_{k}$ to be the power transmitted on the $k^{\text {th }}$ subcarrier. Then we can formulate the constrained optimization problem as

$$
\begin{gathered}
\operatorname{maximize} \sum_{k=1}^{N_{c}} \omega_{k} \\
\text { s.t. C1) } \left.\mathrm{BER}_{k} \leq \mathrm{BER}_{\operatorname{tar}} \text { and } \mathrm{C} 2\right) \sum_{k=1}^{N_{c}} \rho_{k}=P_{\mathrm{T}}
\end{gathered}
$$


where $N_{c}$ is the total number of OFDM subcarriers, $P_{\mathrm{T}}$ is the total available transmit power for all subcarriers and antennas, and $\mathrm{BER}_{k}$ is the average BER evaluated at the $k^{\text {th }}$ subcarrier.

In order to maximize the data rate while achieving a desirable error rate performance without increasing the transmission power, we need to identify a SNR level that is necessary to achieve the target BER before conducting the adaptive bit and power allocation procedure. Therefore, in this section, we will first utilize BER approximation to obtain the required levels of SNR for different signal constellation sizes, and then describe the loading steps of our proposed algorithm.

\subsection{BER Approximation}

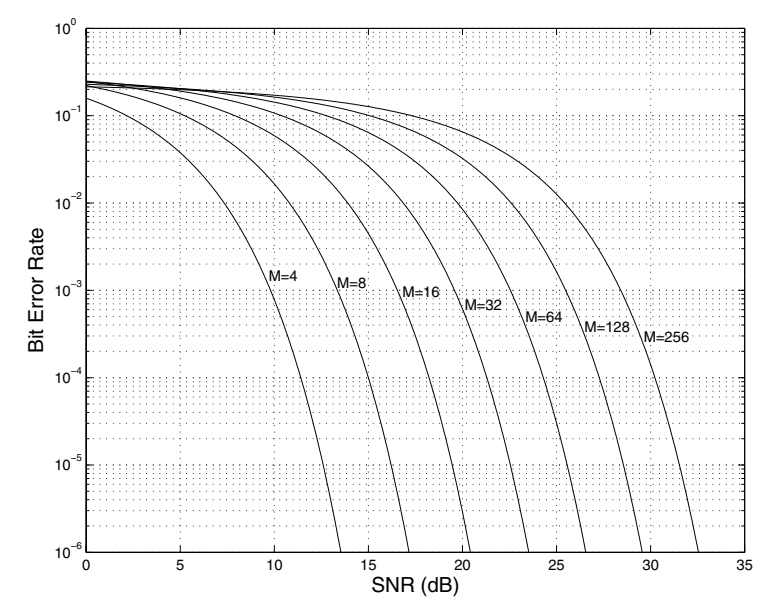

Figure 2. BER approximation for square $M$ ary QAM signals with Gray bit mapping.

Assuming that channel fading gains are perfectly known at the receiver. By examining (3), it is clear that the receive SNR of the $k^{\text {th }}$ subcarrier at the output of MRC has a form

$$
\mathrm{SNR}_{k}=\frac{\varepsilon_{s} \rho_{k}}{\sigma_{k}^{2} N_{t}}\left\|\mathbf{H}_{k}\right\|_{F}^{2}
$$

where $\varepsilon_{s}=\mathcal{E}\left\{\left|x_{k}\right|^{2}\right\}$ is the averaged baseband symbol energy. From here we can define the instantaneous channelto-noise ratio (CNR) at the $k^{\text {th }}$ frequency tone as

$$
\mathrm{CNR}_{k}=\frac{\left\|\mathbf{H}_{k}\right\|_{F}^{2}}{\sigma_{k}^{2}}
$$

For $M$-ary QAM baseband modulated system, the BER is approximated as [9]

$$
\mathrm{BER}_{k} \approx 0.2 \exp \left(\frac{1.6 \mathrm{SNR}_{k}}{2^{\omega_{k}}-1}\right) .
$$

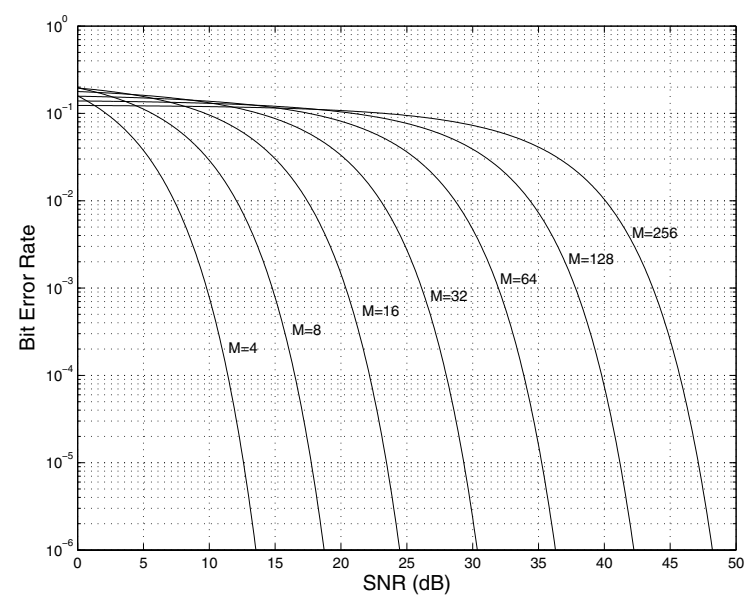

Figure 3. BER approximation for $M$-ary PSK signals with Gray bit mapping.

In Fig. 2, we plotted the BER curves for different QAM constellation size as a function of SNR. Even though this is a generic plot of BER for QAM signals, it can be used for the construction of lookup matrix in our proposed adaptive modulation scheme as we will explain in the next SubSection.

If we rearrange (7) and express it as a function of the required SNR for a target BER and a particular QAM constellation size $\omega$, it will be in the following form

$$
\mathrm{SNR}_{\mathrm{req}}^{\omega} \approx \frac{\left(\ln \left(\frac{\mathrm{BER}_{\mathrm{tar}}}{0.2}\right)\right)(1-M)}{1.6} .
$$

The same approach can be used for $M$-ary PSK signals. The BER equation can be expressed as follows

$$
\mathrm{BER}_{k} \approx \frac{1}{\omega_{k}} \operatorname{erfc}\left(\sqrt{\mathrm{SNR}_{k}} \sin \left(\frac{\pi}{2^{\omega_{k}}}\right)\right)
$$

where $\operatorname{erfc}(\cdot)$ is the standard complementary error function defined as $\operatorname{erfc}(y)=(2 / \sqrt{\pi}) \int_{y}^{\infty} \exp \left(-t^{2}\right) d t$. Again, generic BER curves are plotted for $M$-ary PSK modulated signals as shown in Fig. 3.

\subsection{Algorithm Description}

Let us first describe lookup matrix-ABPA (LM-ABPA) scheme without sub-channel grouping to understand our adaptive allocation process. The algorithm is divided into two major sections: preliminary and loading. Steps involved in the preliminary section need to be performed only at the system initialization stage. Steps in the loading section, however, need to be performed for every new block transmission. 

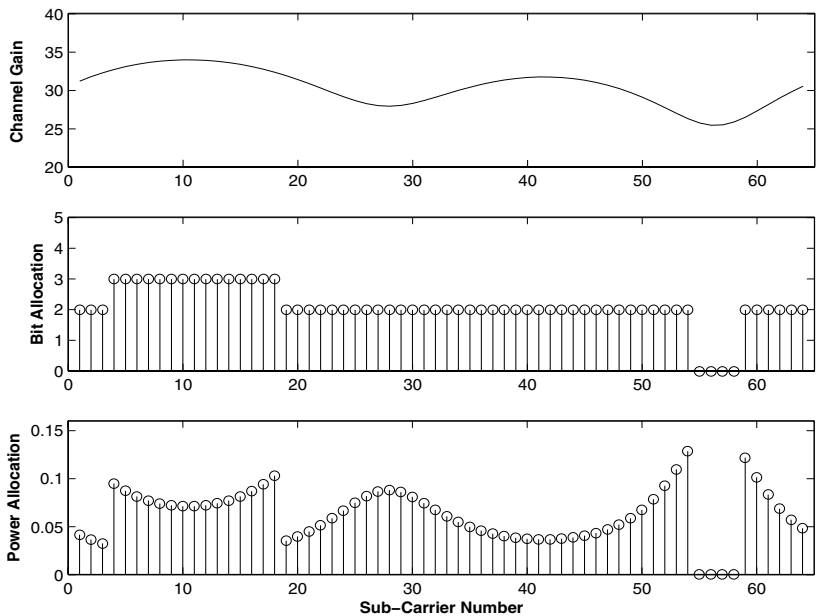

Figure 4. An illustration of the LM-ABPA scheme with $N_{c}=64$ and $P_{\mathrm{T}} / N_{c}$ is normalized to unity. Top: channel-to-noise ratio in dB. Center: bit allocation for 64 subcarriers. Bottom: power allocation for 64 subcarriers.

\section{Preliminary}

Step 1: Determine system $B E R_{\text {tar }}$, e.g. $10^{-3}, 10^{-4}$.

Step 2: $\quad$ Create $\mathrm{SNR}_{\text {req }}=\left[\mathrm{SNR}_{\text {req }}^{1}, \cdots, \mathrm{SNR}_{\text {req }}^{\omega_{\max }}\right]^{T}$ vector for a chosen signal constellation, e.g. PSK, QAM. Elements of this vector can either be found by evaluating (8) or by computer simulation of getting an approximation from Fig. 2 and 3.

\section{Loading}

Step 1: Based on the receiver feedback message of $\mathrm{SNR}_{k}$, estimate $\mathrm{CNR}_{k} \forall k$ by using (6).

Step 2: Construct a Lookup Matrix $\Psi \in \mathbb{R}^{\omega_{\max } \times N_{c}}$ by computing $\mathrm{SNR}_{\text {req }} / \mathrm{CNR}_{k} \forall k$. The column indexes of $\boldsymbol{\Psi}$ correspond to OFDM subcarriers and row indexes correspond to constellation sizes, $\omega$. Thus, the elements of the matrix represent the power required to transmit $\omega$ bits for a particular subcarrier and still satisfy constraint $\mathrm{C} 1$ in (4).

Step 3: To ensure that the least amount of power is always assigned for allocating an addition data bit, we convert $\Psi$ to an incremental power matrix by creating another matrix $\boldsymbol{\Psi}^{\prime}=\mathbf{\Psi}\left(1: \log _{2}\left(\omega_{\max }\right)-1,1\right.$ : $\left.N_{c}\right) \in \mathbb{R}^{\left(\log _{2}\left(\omega_{\max }\right)-1\right) \times N_{c}}$. The conversion of $\Psi$ to an incremental power matrix is then performed as $\boldsymbol{\Psi}=\boldsymbol{\Psi}-\left[\begin{array}{ll}0_{1 \times N_{c}} & \boldsymbol{\Psi}^{\prime}\end{array}\right]_{\log _{2}\left(\omega_{\max }\right) \times N_{c}}^{T}$
Step 4: Add up elements in the lookup matrix $\Psi$ starting from the smallest value. Due to the total power constraint $\mathrm{C} 2$, the sum of elements added must be $\leq P_{\mathrm{T}}$.

Step 5: The final row index and the value in the $k^{\text {th }}$ column corresponds to the number of data bits and power that should be allocated to the $k^{\text {th }}$ subcarrier.

After having LM-ABPA allocation algorithm steps described, it is easy to see that the bit and power allocation algorithm is an algorithm constantly searching for the least amount of power to transmit an extra bit of data ensuring maximum spectral and power efficiency and satisfying both total power and error performance constrains. An example of LM-ABPA result is shown in Fig. 4 with $N_{c}=64$ and $P_{\mathrm{T}} / N_{c}$ is normalized to unity.
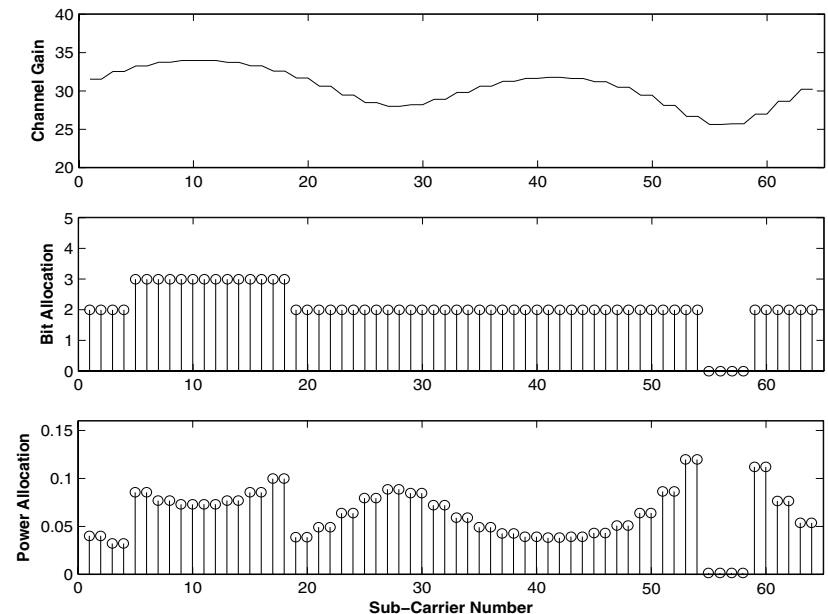

Figure 5. An illustration of LM-SG-ABPA scheme with $N_{c}=64$ and $P_{\mathrm{T}} / N_{c}$ is normalized to unity. Top: channel-to-noise ratio in dB. Center: bit allocation for 64 subcarriers. Bottom: power allocation for 64 subcarriers.

\subsection{Sub-Channel Grouping}

To further reduce the complexity of the proposed scheme, sub-channel grouping based on the channel coherent bandwidth can be used at both the transmitter and the receiver. By grouping subcarriers that are within one coherent bandwidth of the channel, we process multiple subcarriers at a time rather than individually. If the channel coherent bandwidth is equivalent to a bandwidth of $N_{g}$ subcarriers, then the immediate reduction in computation is in a order of $N_{c} / N_{g}$. However, the benefit of sub-channel grouping can only be experienced with the channel being slow fading. In a fast fading environment, i.e., with high Doppler 
spread, LM-ABPA scheme should be used. Fig. 5 depicts the resultant bit and power allocation across subcarriers of the proposed LM-SG-ABPA scheme under the same channel condition as in Fig. 4 and the coherent channel bandwidth is assumed to be equivalent to $N_{g}=2$. It is worth noting that both transmitter and receiver processing simplification from sub-channel grouping will not affect ST block encoding and decoding process. Thus, benefits of diversity gain and coding advantage offered by the STBC technique are retained for the overall system.

\section{Simulation Results}

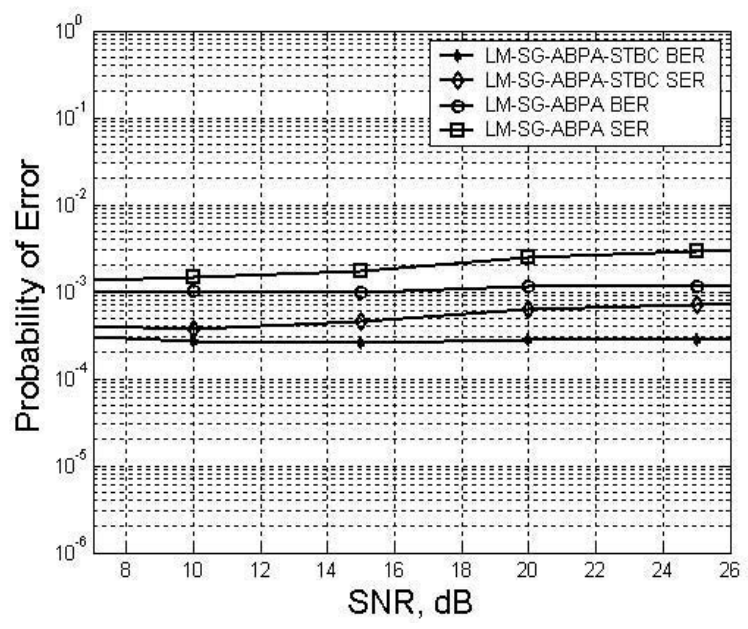

Figure 6. BER and SER performance curves for OFDM systems with the proposed LM-SGABPA-STBC transmission structure and systems with adaptive modulation scheme (LMSG-ABPA) only.

Figure 6 shows error probability performance curves of OFDM systems employing the proposed transmission scheme (LM-SG-ABPA-STBC) and systems with an adaptive modulation (LM-SG-ABPA) scheme only. It is clear that the BER performance of systems with LM-SG-ABPA scheme is constantly maintained at a $\mathrm{BER}_{\operatorname{tar}}$ of $10^{-3}$ and systems with LM-SG-ABPA-STBC scheme achieves a lower BER of approximately $3 \cdot 10^{-4}$. Thus, it demonstrated that the proposed scheme, LM-SG-ABPA-STBC, gives a better performance in both BER and SER. In terms of the spectral efficiency between these two schemes, they would have the same data throughput rate as long as $\mathcal{G} 2$ ST block encoding matrix is used in the proposed transmission structure. As mentioned before, the data rate is halved if $\mathcal{G} 4$ encoding matrix is used.

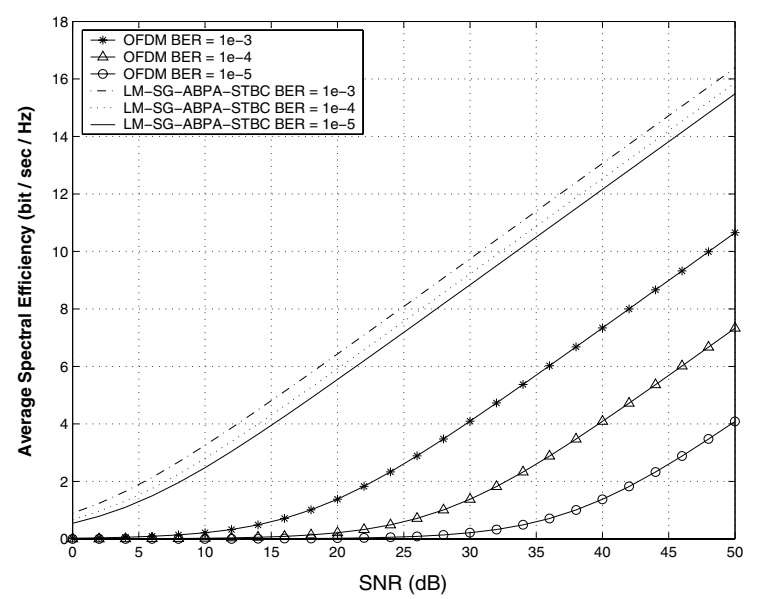

Figure 7. Spectral efficiency of non-adaptive OFDM and LM-SG-ABPA-STBC systems for different values of BER $_{\text {tar }}$.

Now consider the spectral efficiency of OFDM systems employing the proposed transmission structure and compare it with OFDM systems that do not employ this scheme, assuming that the $\mathcal{G} 2$ encoding matrix is used for STBC. By inverting (7), we can express the instantaneous data rate $\omega_{k}$ of QAM modulated signals under a target BER for a given SNR as

$$
\omega_{k}=\log _{2}\left(\frac{1.6 \mathrm{SNR}_{k}}{\ln \left(\frac{\mathrm{BER}_{\mathrm{tar}}}{0.2}\right)}\right) .
$$

For non-adaptive OFDM systems, according to [10], the spectral efficiency can be expressed as

$$
\omega=\log _{2}\left(1+\frac{1.6 \mathrm{SNR}}{\ln \left(\frac{0.2}{\mathrm{BER}_{\mathrm{tar}}}-1\right)}\right) .
$$

Therefore, the mean spectral efficiency $(\mathcal{S})$ can be found by average $\omega$ over all possible channel realizations. In Fig. 7, we showed the average spectral efficiency of non-adaptive OFDM systems and systems employing the proposed LMSG-ABPA-STBC scheme for different BER $\mathrm{tar}_{\text {ar }}$.

The power efficiency of adaptive systems (watts / bit) can be found as

$$
\mathcal{P}_{\mathrm{LM}-\mathrm{SG}-\mathrm{ABPA}}=P_{\mathrm{T}} / \mathcal{S} .
$$

For non-adaptive system, it has the form

$$
\mathcal{P}=P_{\mathrm{T}} / \omega N_{c} .
$$

Figure 8 and 9 show the power efficiency of LM-SG-ABPASTBC and non-adaptive systems, respectively, with $P_{\mathrm{T}} / N_{c}$ 
normalized to unity. It is clear that LM-SG-ABPA-STBC system requires considerably less power per bit than the non-adaptive system for the same BER.

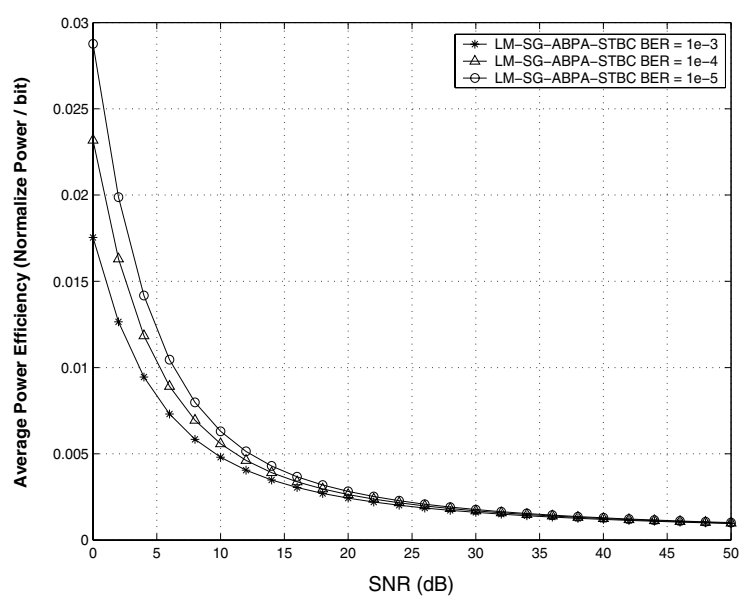

Figure 8. Power efficiency of LM-SG-ABPASTBC systems for different values of $B E R_{\text {tar }}$.

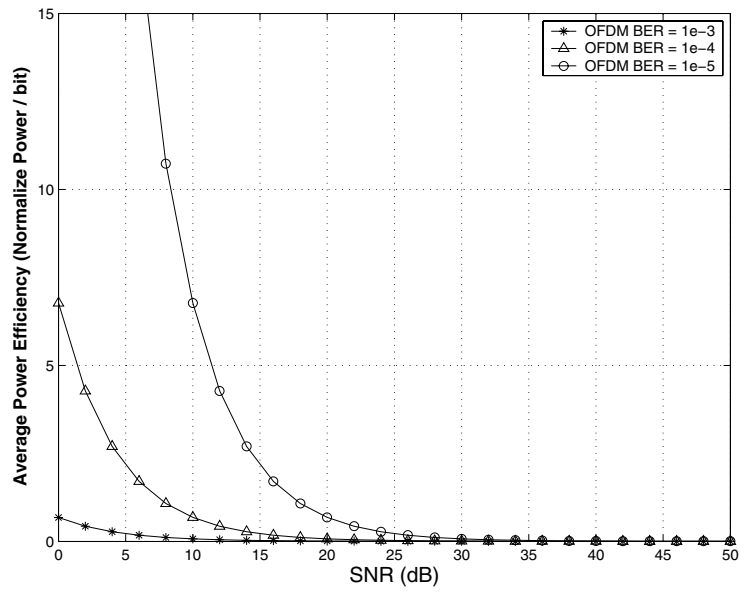

Figure 9. Power efficiency of non-adaptive OFDM systems for different values of $B R_{t a r}$.

\section{Conclusions}

In this paper a new transmission structure that combines adaptive modulation with STBC for MIMO-OFDM systems is proposed. With an effective utilization of receiver feedback message, optimum allocation of information bits and transmit power to subcarriers is performed and the spectral and power efficiency of space-time block encoded OFDM is greatly improved. To simplify the overall computation complexity of the system, sub-channel grouping is incorporated within the adaptive modulation scheme to reduce the lookup matrix size and the amount of feedback signalling. Simulation results demonstrated that the proposed LM-SGABPA-STBC scheme achieves a significantly higher spectral and power efficiency over systems that do not employ this scheme and at the same time gives a lower BER than the target BER of the system.

\section{References}

[1] G. J. Foschini and M. J. Gans, "On limits of wireless communications in a fading environment when using multiple antennas," Wireless Pers. Commun., vol. 6, no. 3, pp. 311335, Mar. 1998.

[2] I. E. Telatar, "Capacity of multi-antenna Gaussian channels," Eur. Trans. Tel., vol. 10, no. 6, pp. 585-595, Nov./Dec. 1999.

[3] V. Tarokh, N. Seshadri, and A. R. Calderbank, "Space-time codes for high data rate wireless communication: Performance criterion and code construction," IEEE Trans. Inform. Theory, vol. 44, no. 2, pp. 744-764, Mar. 1998.

[4] V. Tarokh, H. Jafarkhani, and A. R. Calderbank, "Spacetime block coding for wireless communications: Performance results," IEEE J. Select. Areas in Commun., vol. 17, pp. 451-460, Mar. 1999.

[5] P. S. Chow, J. M. Cioffi, and J. A. C. Bingham, ”A practical discrete multitone transceiver loading algorithm for data transmission over spectrally shaped channels," IEEE Trans. Commun., vol. 43, no. 2/3, pp. 773-775, Feb./Mar. 1995.

[6] A. Czylwik, "Adaptive OFDM for wideband radio channels," in Proc. IEEE GLOBECOM, 1996, pp. 713-718.

[7] T. Keller and L. Hanzo, "Adaptive multicarrier modulation: A convenient framework for time-frequency processing in wireless communications," Proc. IEEE, vol. 88, no. 5, pp. 611-640, May 2000.

[8] K. H. Lin and Z. M. Hussain, "Adaptive bit and power allocation with sub-channel grouping for OFDM systems," in $3 r d$ Workshop on the Internet, Telecommunications and Signal Processing WITSP'2004, Dec. 2004.

[9] P. S. Chung and A. J. Goldsmith, "Degrees of freedom in adaptive modulation: A unified view," IEEE Trans. Commun., vol. 49, no. 9, pp. 1561-1571, Sep. 2001.

[10] S. Ye, R. S. Blum, and L. J. Cimini Jr., "Adaptive modulation for variable-rate OFDM systems with imperfect channel information," in Proc. IEEE VTC Spring, 2002, pp. 767-771. 